\title{
Clinical and imagistic correlations in the inflammatory pathology of nasosinusal cavities
}

\author{
Emilia Diaconu
}

Imaging Center, "Regina Maria" Private Health Care Network, Bucharest, Romania

\section{ABSTRACT}

This article reviews the pathophysiology and the clinical and imaging diagnosis of rhinosinusal inflammations.

Chronic sinusitis usually develops after an incompletely treated acute sinusitis.

Complications occur through fungal superinfection, osteomyelitis, extension of the inflammatory process in the orbit or adjacent meningoencephalic structures.

The imaging investigation is indicated as the symptoms get worse, for the elucidation of sinus contents, for establishing the therapeutic protocol, for identifying and finding solutions to solve nearby complications.

KEYWORDS: nasal cavities, paranasal sinuses, rhinosinusal inflammatory pathology, computed tomography, magnetic resonance.

\section{INTRODUCTION}

The inflammatory sinonasal disease is one of the most common human disorders. Almost everyone has experienced episodes of sinonasal inflammation, of viral, bacterial, allergic, vasomotor or reactive cause.

Imaging investigations such as computed tomography (CT) and magnetic resonance imaging (MRI) bring information even more useful as the stage of the disease is more advanced ${ }^{1,2}$.

\section{RHINOSINUSAL INFLAMMATORY PATHOLOGY}

Viral common cold is rarely investigated imagistically, is self-limited; it remains at the level of viral rhinitis and minimal sinusitis. If images are obtained, they usually show the thickening of the nasal mucosa, the swelling of nasal turbinates and little or no thickening of the sinus mucosa. Nasal secretions drain into the upper airway causing coughing.
Bacterial sinusitis occurs when the enlargement of nasal turbinates or edema of the sinus mucosa causes obstruction of sinus ostia. This causes the decrease in intrasinusal oxygen pressure, the alteration of normal bacterial flora and allows the development of pathological flora from oro-respiratory secretions, depending on the patient's immune status ${ }^{3}$ (Figure 1).

Also, for the maxillary sinus, $10-20 \%$ of the disorders are secondary to infections and dental extractions - odontogenic sinusitis.

The CT examination at this stage reveals the edema of the submucosa, the mucous membrane lining, the alveolar bone grafting or the intrusion of other sinus bone walls by expansion or erosion (Figure 2).

The secretions accumulated intrasinusally are evaluated by magnetic resonance, their signal varying depending on the protein content / degree of desiccation, and their description is relevant to the surgeon since, the thicker they are, the harder they can be drained and may change the surgical approach ${ }^{4}$.

The expanded sinus, increased in volume, completely full, with fluid / parafluid content, with edematous mucosa, with some walls thinned by atrophy due to pressure, characterizes a mucocele (Figure 3 ). 


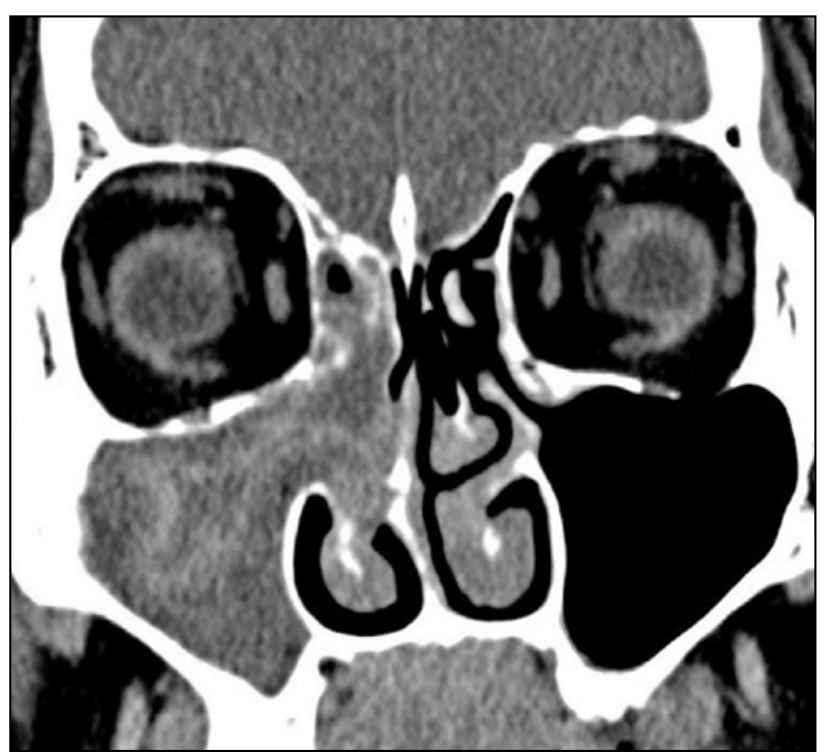

Figure 1 Coronal CT scan - acute bacterial right maxillary sinusitis.

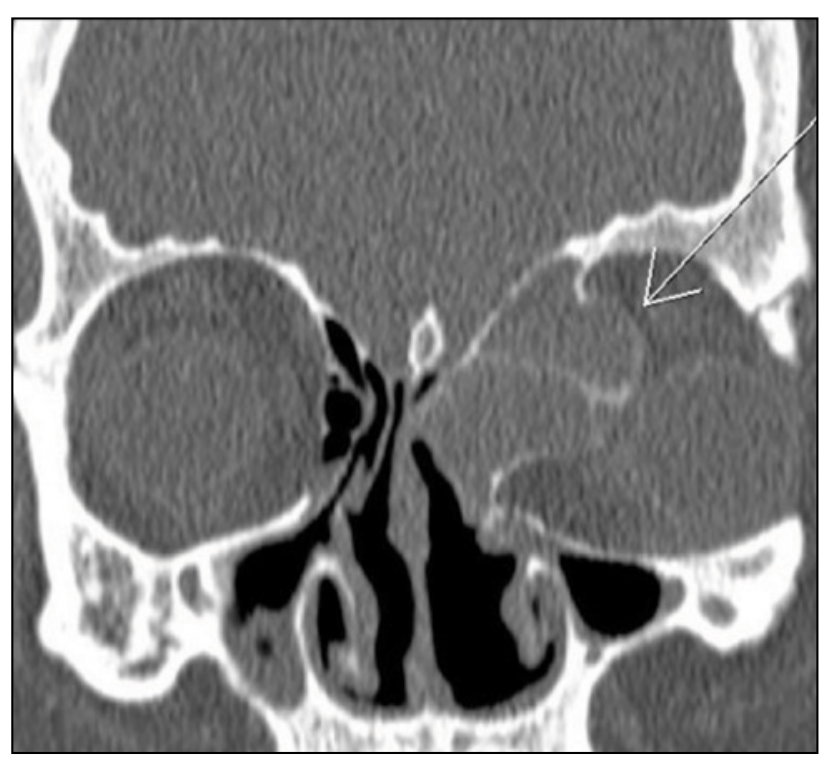

Figure 3 Coronal CT scan - left ethmoidal mucocele, prolapsed intraorbitally.

If it clinically associates sinus pain or headache, we talk about a mucopyocele ${ }^{4}$.

Chronic sinusitis is a result of acute inflammation dragging on or of repeated episodes of acute or subacute sinusitis. In this context, the sinus mucosa becomes atrophic, sclerotic or hypertrophic polypoid.

Secretions accumulated in the chronically inflamed sinus may remain fluid, may become desiccated / condensed, becoming hyperdense on CT and with modified MRI signal, can be over-infected with fungi or can grow in volume progressively, developing a mucocele.

The bone wall of a chronically inflamed sinus becomes thickened and reactively osteo-condensed (Figure 4$)^{5}$.

Sinus and nasal polyposis is most commonly associated with an allergic etiology.

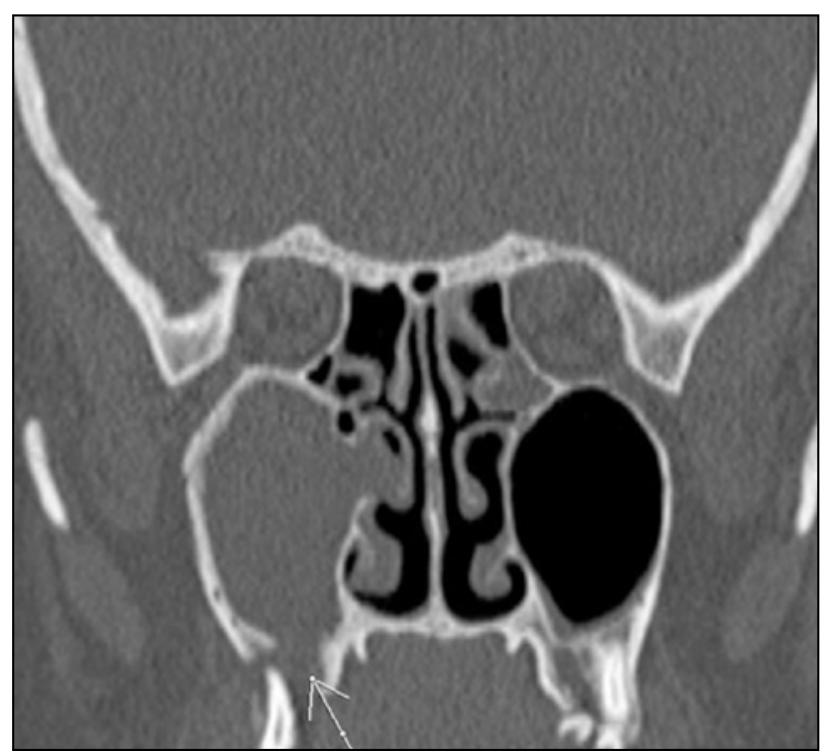

Figure 2 Coronal CT scan - right maxillary odontogenic sinusitis, oroantral communication.

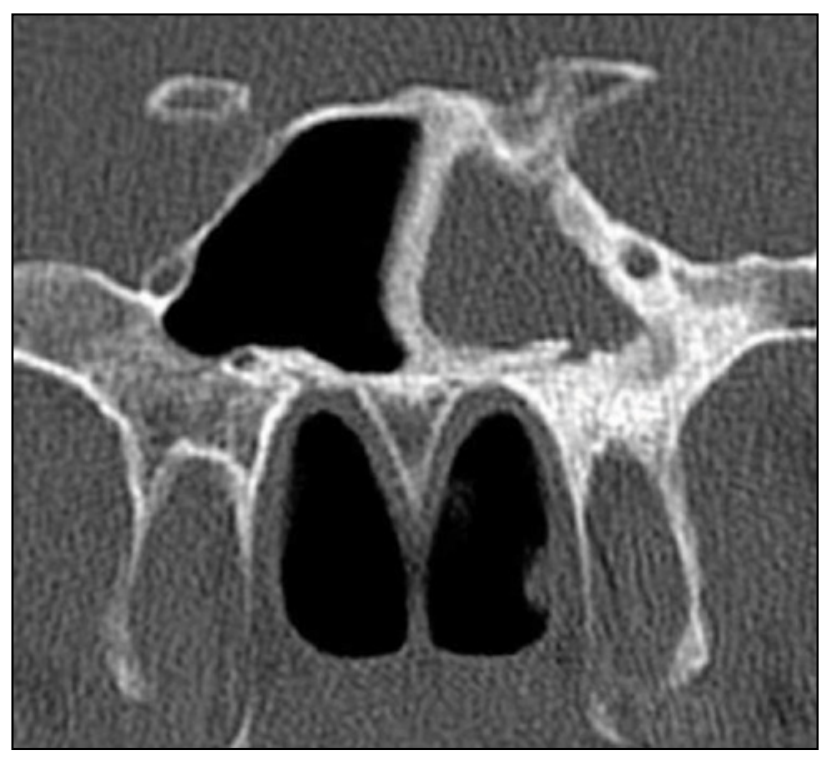

Figure 4 Coronal CT scan - chronic left sphenoid sinusitis, thickening and osteo-condensation of the sinus wall.

In the case of bacterial sinusitis, the accompanying polyp is rare, and is usually unique ${ }^{6}$ (Figure 5).

Fungal sinusitis has 4 clinical-pathological types: 1 . acute fulminant invasive disease (in immunocompromised patients / haematological malignancies); 2. chronic invasive infection (granulomatous) - frequently in diabetics; 3 . non-invasive fungal colonization (mycetoma, "fungus ball") - usually due to changes in the local microclimate, surgery, radiotherapy, endodontic treatment, and, anecdotally, smoking marijuana; 4. allergic fungal sinusitis (eosinophilic sinusitis - predisposed individuals develop extreme hypersensitivity, eosinophil-mediated reactions to ubiquitous fungi) ${ }^{4}$. 


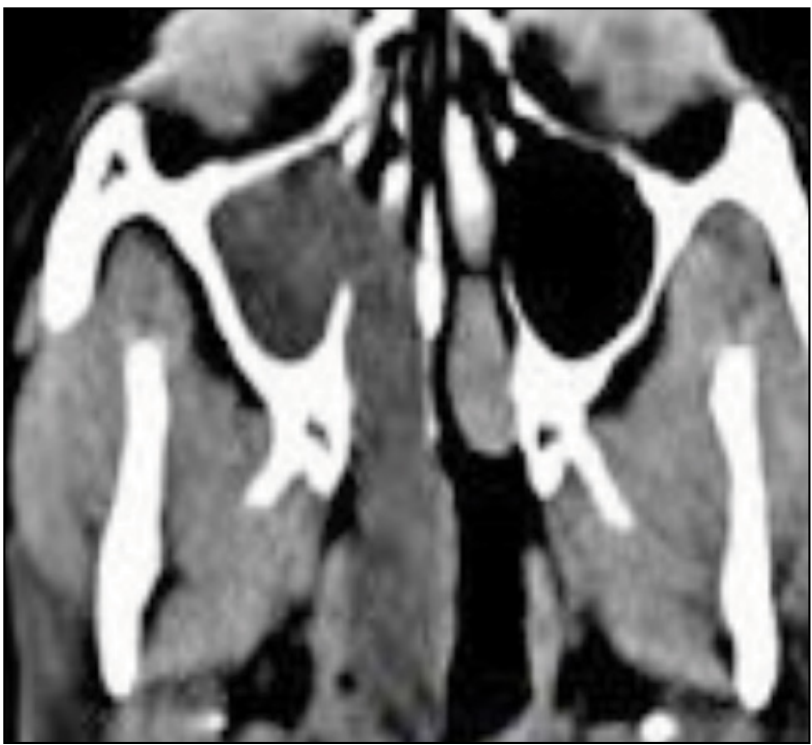

Figure 5 Axial CT scan - antrochoanal polyp - hypodense mass in the right maxillary sinus, extending into the nasal cavity and reaching the cavum.

The imaging exam detects volume expansion and the heterogeneous sinus opacification, the CT scan detects hyperdensity and the typical MRI the T2 nonsignal and bone erosion (Figure $6 \mathrm{a}, \mathrm{b}$ ).

\section{Intrasinusal foreign bodies}

- non-metallic (beads, buttons, peanuts, etc.), cannot be distinguished on CT if they are radiotransparent;

- metallic (including root filling material and dental implants), easily visible on CT.

Moreover, if they have been in the sinus for a long time, they can serve as nidus for calcareous deposits (rhinoliths, sinoliths) and can trigger neighbouring inflammatory reactions or by the obstructive effect ${ }^{4}$.

\section{COMPLICATIONS OF NASOSINUSAL INFLAMMATIONS AFFECTING ADJACENT AREAS}

Intracranial dissemination of the infection in the frontal, sphenoid and ethmoid sinuses should not be associated with a large bone destruction of the sinus wall, because it can be spread through small emissary veins, which are not visible on CT or MRI.

The suspicion of intracranial spread of infection requires a brain MRI exam with contrast (Figure 7). It may reveal meningitis, epidural abscess, subdural abscess, brain abscess.

Orbital and pre-septal inflammation includes orbital fat edema, preseptal cellulitis, preseptal abscess, orbital cellulitis, orbital subperiosteal abscess, intraorbital abscess, cavernous sinus thrombosis (Figure 8).

Orbital cellulitis may appear slightly secondary to bacterial sinusitis due to the thin lamina papyracea and the absence of valves in the anterior and posterior ethmoidal veins, as well as the direct intrusion of the orbital floor from the maxillary sinus or of the orbital roof from the supraorbital ethmoidal cells or from the infected frontal sinus.

Also, the contiguity of the lacrimal drainage system with anterior ethmoidal cells can lead to pseudodacryocystitis, in which case it is necessary to clarify the cause, which changes the therapeutic protocol ${ }^{4}$.

Osteomyelitis can complicate the aggressive bacterial or fungal sinusitis, or that occurring on an irradiated facial region ${ }^{7}$. The CT exam detects bone rarefaction, bone sequestra forming, thickening and reactive sclerosis of the bone, bone fragmentation.
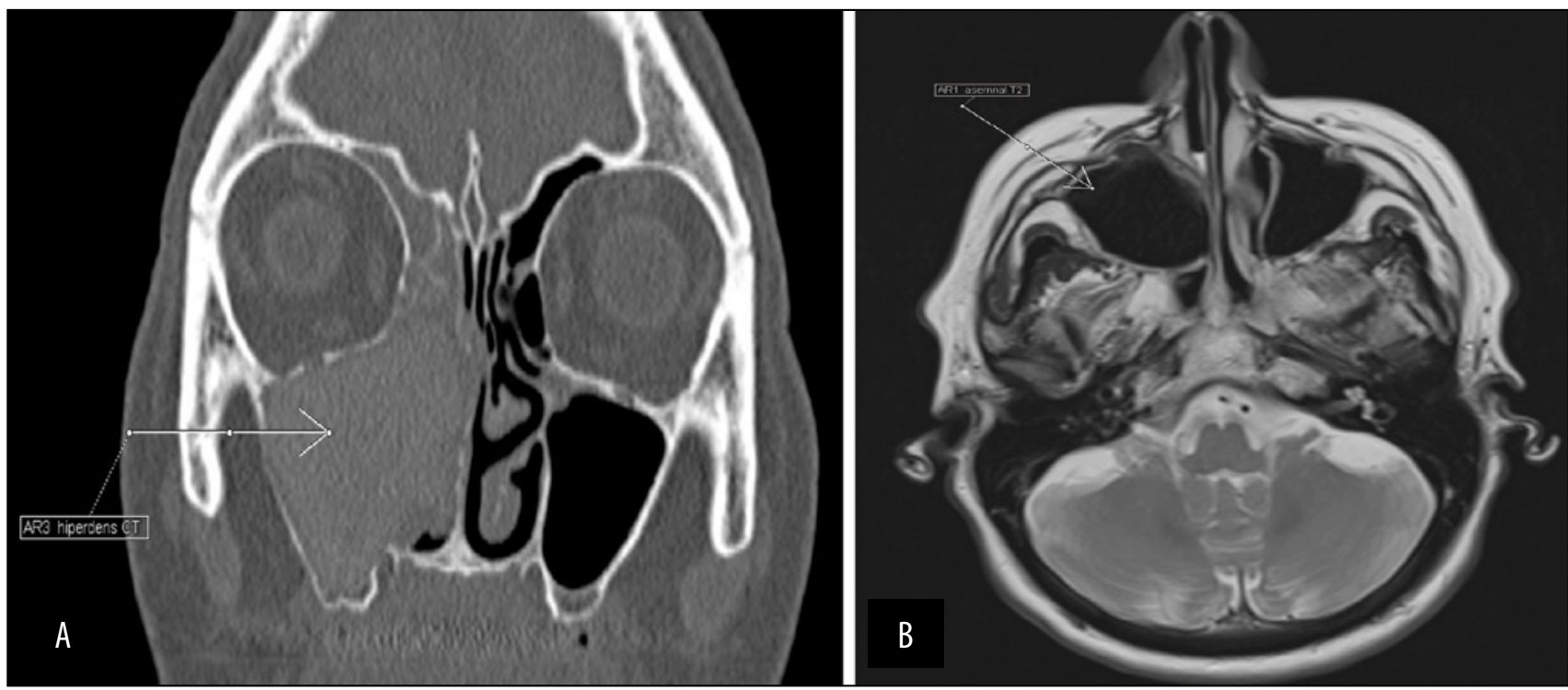

Figure 6 (A) Coronal CT scan - fungal sinusitis, erosion of bone walls, hyperdense sinus contents; (B) T2 MRI axial section - fungal sinusitis, signal void creating a similar appearance between the right maxillary sinus infected with fungi and the air in the left maxillary sinus. 


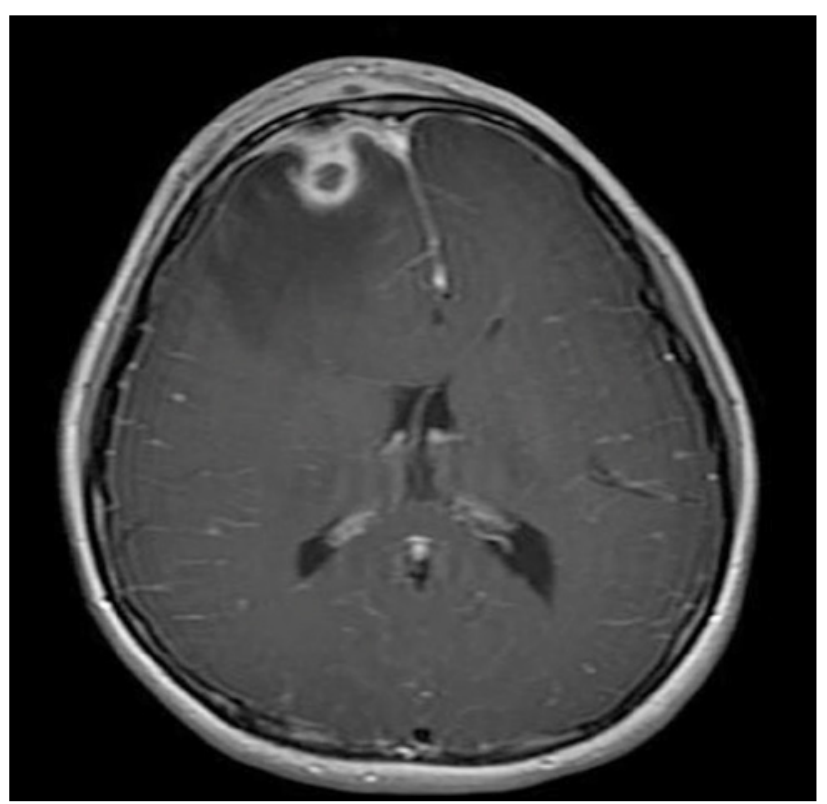

Figure 7 MRI exam - intracerebral abscess secondary to frontal sinusitis.

\section{DISCUSSIONS}

The imaging investigation of this pathology should preferably begin with the CT scan without contrast. It allows to distinguish between bone, mucosal thickening, fluid retention, air.

During the magnetic resonance exam, the desiccated chronic secretion may have a signal void and may be confused with the air.

If the material accumulated in the sinus has CT hyperdensity, it can be made up of desiccated collections, mycetoma / fungal disease or haemorrhage.

The contact area of a possible tumor can no longer be distinguished on CT from these native hyperdense structures. This is where the superiority of the MRI exam becomes obvious.

If a contrast substance is administered and the mucosa is loaded linearly, with thickening of the edematous submucosa, non-iodophil, subjacent, then it is inflamed. If it is thick but does not load, then it is mucosal scarring / fibrosis, without active inflammation.

The intrasinusal polyp and the retention cyst which does not completely occupy the sinus have the same CT and MRI aspect.

Fluid retention can generate a full sinus, with no change in volume.

The expanded sinus is considered a mucocele. If it is not over-infected, it has a fine, uniform border, with contrast-loaded mucosa. If it is over-infected (mucopyocele), the mucosa is irregularly thickened and loaded more heterogeneously.

Neighbouring complications of sinus inflammation are detected by the head CT and MRI exams.

The imaging investigation is indicated as the symptoms persist, to elucidate the sinus contents, establish-

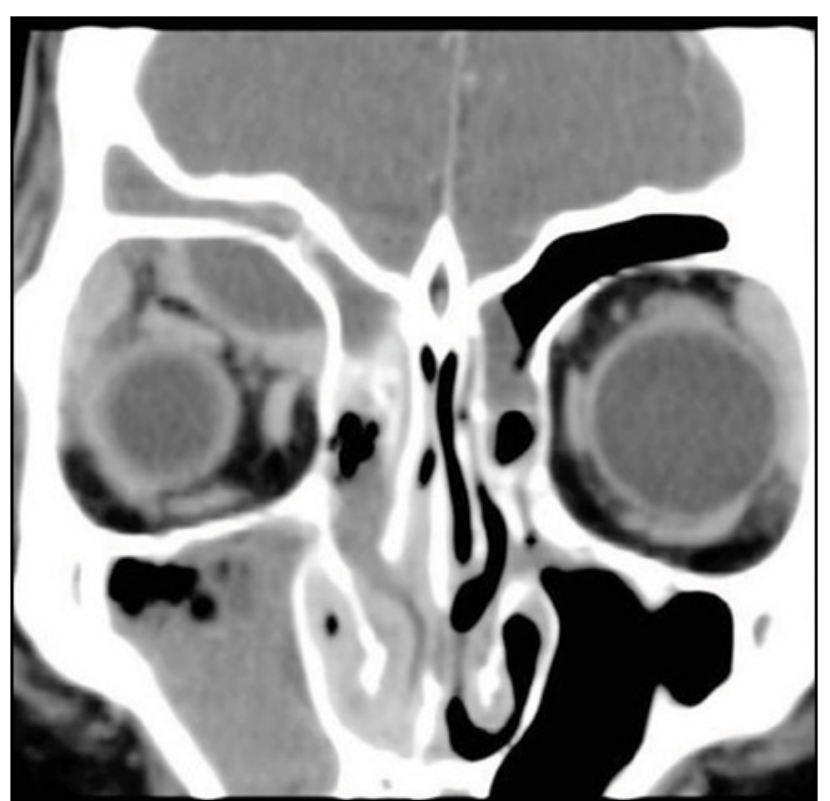

Figure 8 Coronal CT - right superomedial orbital subperiostal abscess.

ing the therapeutic protocol, identifying and finding solutions to address neighbouring complications ${ }^{8}$.

\section{CONCLUSIONS}

Computed tomography (CT) scanning is the examination of choice in sinusitis, particularly in cases of chronic sinus disease, providing excellent detail of the sinus anatomy. However, CT is usually not useful in acute sinusitis, as diagnosis in acute cases is primarily based on clinical findings. Good anatomic definition is desirable before the surgical intervention.

Coronal CT imaging is the preferred initial procedure. Bone-window views provide excellent resolution and good definition of the complete ostiomeatal complex and other anatomic details that play a role in sinusitis. In addition, the coronal view is best correlated with findings from sinus surgery, with anatomy and pathology visualized in a plane almost identical to that seen by the endoscopist.

CT provides an excellent anatomic display of softtissue attenuation. This depiction includes fluid levels and polypoid masses within the normally air-filled cavities of the sinuses, nasal cavity and postnasal space. Most important, the disease extending beyond the bony perimeters of the sinuses into the adjacent soft tissue of the orbit, brain and infratemporal fossa can be imaged.

Magnetic resonance imaging (MRI) is generally reserved for the evaluation of any complications of local sinus infections, particularly suspected intracranial extension. The ability to image in any plane is a considerable advantage in $\mathrm{MRI}^{9}$. 
Conflict of interest: The authors have no conflict of interest.

Contribution of authors: All authors have equally contributed to this work.

\section{REFERENCES}

1. Hasso AN. Diagnostic Imaging of the Head and Neck: MRI with CT \& PET Correlations. LWW, Philadelphia, USA; 2011.

2. Czerny C. Functional and sectional anatomy of the nose and paranasal sinuses. Erasmus Course on Magnetic Resonance Imaging Head \& Neck; 2010 .

3. Bell DJ, Gaillard F, et al. Acute sinusitis. Radiopaedia. Available from: https://radiopaedia.org/articles/acute-sinusitis. [Accessed January 3rd 2018].
4. Som PM, Curtin HD. Head and Neck Imaging. Fifth Edition. Volume 1. Mosby; 2011.

5. Knipe H, Weerakkody Y et al. Chronic sinusitis. Radiopaedia. Available from: https://radiopaedia.org/articles/chronic-sinusitis. [Accessed January 3rd 2018].

6. Caruana NB, Makariou E. Antrochoanal Polyp. Diagnostic Imaging. 2012. Available from: http://www.diagnosticimaging.com/articles/ antrochoanal-polyp-0/page/0/2. [Accessed January 3rd 2018].

7. Bickle I, Gaillard F, et al. Pott puffy tumour. Radiopaedia. Available from: https://radiopaedia.org/articles/pott-puffy-tumour. [Accessed January 3rd 2018].

8. Momeni AK, Roberts CC, Chew FS. Imaging of chronic and exotic sinonasal disease: review. AJR Am J Roentgenol. 2007;189(6 Suppl):S35-45 DOI: 10.2214/AJR.07.7031

9. Ramanan RV, Khan AN, Sabih D. Sinusitis Imaging. Medscape. Updated: January 03, 2016. Available from: https://emedicine.medscape.com/ article/384649-overview. [Accessed January 3rd 2018]. 